Recent Developments in Gravity: Proceedings of the 10th Hellenic Relativity Conference, World Scientific (2003)

\title{
GEOMETRICAL ASPECTS OF AN INTEGRABLE NONLINEAR EQUATION OF THE SCHWARZIAN TYPE
}

\author{
A. TONGAS*
}

Department of Mathematics, University of Patras, 26500 Patra, Greece E-mail: tasos@math.upatras.gr

We show how to construct four-dimensional anti-self-dual conformal structures from solutions of a parameter family of partial differential equations which generalize the Ernst equation of General Relativity. Anti-self-dual vacuum metrics may be obtained as well by restricting appropriately the values of the parameters.

\section{Introduction}

Consider the following Lagrangian ${ }^{1}$

$$
\mathcal{L}[U]=(v-u) \frac{U_{, u v}^{2}}{U_{, u} U_{, v}}+\frac{1}{v-u}\left(\alpha^{2} \frac{U_{, u}}{U_{, v}}+\beta^{2} \frac{U_{, v}}{U_{, u}}\right),
$$

where $U$ is a scalar function of $(u, v)$ and $\alpha, \beta$ are parameters. Varying the function $U$ we get the fourth order partial differential equation (PDE)

$$
\partial_{u}\left(\mathcal{A}_{, v}+\frac{\mathcal{A}^{2}}{\rho} U_{, v}+\alpha \frac{\mathcal{A}}{\rho}\right)+\partial_{v}\left(\mathcal{A}_{, u}+\frac{\mathcal{A}^{2}}{\rho} U_{, u}-\beta \frac{\mathcal{A}}{\rho}\right)=0,
$$

where

$$
\mathcal{A}=\frac{1}{2}\left((v-u) \frac{U_{, u v}}{U_{, u} U, v}+\frac{\beta}{U_{, u}}-\frac{\alpha}{U_{, v}}\right) .
$$

The above parameter family PDE admits a linear representation (Lax pair) by introducing two auxiliary variables $P$ and $R$ from the relation $\mathcal{A}=P-R$. The Lax pair is given by the linear operators in a parameter $\lambda \in \mathbb{C P}^{1}$

$$
L=\left(u \partial_{u}-A\right)-\lambda \partial_{u}, \quad M=\left(v \partial_{v}-B\right)-\lambda \partial_{v},
$$

where

$$
A=\left(\begin{array}{cc}
\beta-P U_{, u} & U_{, u} \\
P\left(n-P U_{, u}\right) & P U_{, u}
\end{array}\right), \quad B=\left(\begin{array}{cc}
-R U_{, v} & U_{, v} \\
R\left(m-R U_{, v}\right) & R U_{, v}-\alpha
\end{array}\right)
$$

*Work supported by University of Patras research project C. Karatheodoris, 2785/2001. 
The commutativity condition $[L, M]=0$ for every value of $\lambda$ leads to the following system of PDEs

$$
\begin{aligned}
P_{, v} & =\frac{P-R}{u-v}\left(\alpha+(P-R) U_{, v}\right), \\
R_{, u} & =\frac{P-R}{u-v}\left(\beta-(P-R) U_{, u}\right), \\
U_{, u v} & =\frac{1}{u-v}\left(\beta U_{, v}-\alpha U_{, u}-2(P-R) U_{, u} U_{, v}\right) .
\end{aligned}
$$

By decoupling the dependent variables one finds that $U$ satisfies equation (2), while the auxiliary variables $P, R$ satisfy the same forth order PDE and defer only by specific changes of the parameters $(m, n)$. Thus, the above system may be regarded as representing an involution form for a PDE which was called the regular PDE (RPDE) by its inventors ${ }^{1}$. Its significance stems from the fact that it encodes the entire hierarchy of KdV equations which is the prototype of the soliton equations. By applying appropriate reality conditions on the generally complex valued variables and parameters, one may obtain from the system (6)-(8) the hyperbolic Ernst equation of General Relativity in the presence of neutrino fields ${ }^{2}$.

Here we reveal yet another link of the RPDE with geometry and physics by presenting a method of constructing anti-self-dual metrics in $2+2$ dimensions from its solutions. This is the subject of the last section. In the following two sections we briefly recall some basic definitions regarding the anti-self-dual (ASD) Yang-Mills equations and ASD curved spacetimes. For a comprehensive exposure on the subject we refer to the monographs by Mason and Woodhouse ${ }^{3}$ and by Ward and Wells ${ }^{4}$.

\section{The anti-self-dual Yang-Mills equations}

Consider the four dimensional complex Minkowski spacetime $\mathbb{C M}$ with double null coordinates $x^{a}=(w, z, \tilde{w}, \tilde{z})$ and metric

$$
\mathrm{d} s^{2}=\mathrm{d} z \otimes \mathrm{d} \tilde{z}+\mathrm{d} \tilde{z} \otimes \mathrm{d} z-\mathrm{d} w \otimes \mathrm{d} \tilde{w}-\mathrm{d} \tilde{w} \otimes \mathrm{d} w .
$$

The connection $\Phi$ on a rank $r$ vector bundle over $\mathbb{C}^{4}$ is a $\mathbf{g l}(r, \mathbb{C})$-valued 1 -form on $\mathbb{C M}$ and the corresponding curvature $\mathrm{F}$ is the $\operatorname{gl}(r, \mathbb{C})$-valued 2-form $\mathrm{F}=\mathrm{d} \Phi+\Phi \wedge \Phi$.

The connection $\Phi$ is said to be anti-self-dual if and only if $\mathrm{F}$ is Hodge anti-self-dual with respect to the metric (9) i.e. $\mathrm{F}=-* \mathrm{~F}$. Choosing an orientation the anti-self-duality condition is equivalent to the following system of equations

$$
\mathrm{F}_{z w}=0, \quad \mathrm{~F}_{z \tilde{z}}-\mathrm{F}_{w \tilde{w}}=0, \quad \mathrm{~F}_{\tilde{z} \tilde{w}}=0,
$$


which are called the anti-self-dual Yang-Mills (ASDYM) equations. Equations (10) are equivalent to the commutativity of the following linear operator $^{5}$

$$
\widetilde{L}=\partial_{w}+\Phi_{w}-\zeta\left(\partial_{\tilde{z}}+\Phi_{\tilde{z}}\right), \quad \widetilde{M}=\partial_{z}+\Phi_{z}-\zeta\left(\partial_{\tilde{w}}+\Phi_{\tilde{w}}\right),
$$

for every value of the parameter $\zeta \in \mathbb{C P}^{1}$.

\section{Anti-self-dual metrics}

Let $\mathcal{M}$ be a four dimensional manifold with a local holomorphic coordinate basis $x^{a}=\left(x^{1}, x^{2}, x^{3}, x^{4}\right)$ and a holomorphic metric $g$ with respect to $x^{a}$. $(\mathcal{M}, g)$ is said to be anti-self-dual if and only if the Weyl tensor is Hodge anti-self-dual with respect to $g$.

Since the Weyl tensor is a conformally invariant object, the above ASD condition is defined up to conformal transformation $g \mapsto e^{2 \sigma(p)} g$, where $\sigma$ is a holomorphic function on $\mathcal{M}$. Such transformations define an equivalence relation among the set of metrics on $\mathcal{M}$ and the equivalence class $(M,[g])$ is called $A S D$ conformal structure.

A convenient way of constructing ASD metrics is as follows ${ }^{6,7}$. Suppose that we choose the local basis $\left\{\mathbf{e}_{i}\right\}$ of $T \mathcal{M}$ to be a null tetrad such that $g\left(\mathbf{e}_{1}, \mathbf{e}_{4}\right)=-g\left(\mathbf{e}_{2}, \mathbf{e}_{3}\right)$ and let $\left\{\theta^{i}\right\}$ be the dual basis. Define

$$
\ell=\mathbf{e}_{3}-\lambda \mathbf{e}_{1}, \quad m=\mathbf{e}_{4}-\lambda \mathbf{e}_{2},
$$

where $\lambda \in \mathbb{C P}^{1}$. If $\ell$ and $m$ commute for every value of $\lambda$ then the metric $g$ given by the line element

$$
\mathrm{d} s^{2}=\theta^{1} \otimes \theta^{4}+\theta^{4} \otimes \theta^{1}-\theta^{2} \otimes \theta^{3}-\theta^{3} \otimes \theta^{2},
$$

is a representative of an ASD conformal structure.

Moreover, if the vector fields $\mathbf{e}_{i}$ are divergence free with respect to a 4 -form $\nu$ on $\mathcal{M}$ then the rescaled metric $\Lambda g$ has vanishing Ricci tensor, where $\Lambda=\nu\left(\mathbf{e}_{1}, \mathbf{e}_{2}, \mathbf{e}_{3}, \mathbf{e}_{4}\right)$.

\section{From solutions of the RPDE to ASD metrics}

Suppose that the ASD connection $\Phi$ on a rank 2 vector bundle over $\mathbb{C M}$ remains invariant under the action of the two dimensional group of transformations generated by the following conformal Killing vector fields

$$
X=w \partial_{w}+\tilde{z} \partial_{\tilde{z}}, \quad Y=z \partial_{z}+\tilde{w} \partial_{\tilde{w}} .
$$


Let $u=\frac{w}{\tilde{z}}, v=\frac{z}{\tilde{w}}$ be the invariant coordinates and impose gauge conditions such that $\Phi_{z}=0, \Phi_{w}=0$. The remaining invariant components of the connection have the form

$$
\Phi_{\tilde{z}}=\frac{1}{\tilde{z}} A(u, v), \quad \Phi_{\tilde{w}}=\frac{1}{\tilde{w}} B(u, v) .
$$

The reduced ASDYM equations are equivalent to the commutativity of the following operators

$$
L=\left(u \partial_{u}-A\right)-\lambda \partial_{u}, \quad M=\left(v \partial_{v}-B\right)-\lambda \partial_{v},
$$

for every value of $\lambda$. Choosing the matrices $A$ and $B$ from (5), the relation $[L, M]=0$ is equivalent to the equations (6)-(8). In this sense the RPDE is a two-dimensional reduction of the ASDYM equations ${ }^{8}$.

As such, one may reinterpret the ASD condition on the curvature F as the ASD condition on the conformal curvature $C$, by employing the switch map ${ }^{3}$. The explicit link is provided by observing that equations (6)-(8) are equivalent to the commutativity of the linear operators given by (16), and the commutativity of the linear vector fields $\ell, m$ in $\lambda$ given by (12) is sufficient for a four dimensional metric to be ASD. To be more specific, first we make the identification $\mathbf{e}_{1}=\partial_{u}, \mathbf{e}_{2}=\partial_{v}$. Next, using the infinitesimal representation of the natural action of $\mathbf{G L}(2, \mathbb{C})$ on $\mathbb{C}^{2}$, one may represent the matrices $A, B$ as vector fields on $\mathbb{C}^{2}$ with coordinates $(p, q)$. Explicitly, an arbitrary element $M \in \operatorname{gl}(2, \mathbb{C})$ may by represented by a vector field $\mathcal{V}_{M} \in \operatorname{diff}\left(\mathbb{C}^{2}\right)$ as follows

$$
M=\left(\begin{array}{ll}
a_{11} & a_{12} \\
a_{21} & a_{22}
\end{array}\right) \longleftrightarrow \mathcal{V}_{M}=\left(a_{12} q-a_{11} p\right) \partial_{p}+\left(a_{21} p-a_{22} q\right) \partial_{q} .
$$

Using the above correspondence it is straightforward to represent the operators (16) by vector fields of the form (12) respectively, where

$$
\begin{array}{ll}
\mathbf{e}_{1}=\partial_{u}, & \mathbf{e}_{3}=u \partial_{u}+\beta p \partial_{p}+f_{, q} \partial_{p}-f_{, p} \partial_{q}, \\
\mathbf{e}_{2}=\partial_{v}, & \mathbf{e}_{4}=v \partial_{v}-\alpha q \partial_{q}+g, q \partial_{p}-g, p \\
\partial_{q}
\end{array}
$$

In the above vector fields the scalar functions $f, g$ are given by the following second degree homogeneous polynomials in $p, q$ with $(u, v)$ dependent coefficients

$$
f=-\frac{1}{2} U_{, u}(q+P p)^{2}+\frac{\beta}{2} P p^{2}, \quad g=-\frac{1}{2} U_{, v}(q+R p)^{2}+\frac{\alpha}{2} R p^{2} .
$$

The dual basis is given by the following 1 -forms

$$
\begin{array}{ll}
\theta^{1}=\mathrm{d} u-u \theta^{3}, & \theta^{3}=\left(\left(\alpha q+g_{, p}\right) \mathrm{d} p+g_{, q} \mathrm{~d} q\right) / \Delta, \\
\theta^{2}=\mathrm{d} v-v \theta^{4}, & \theta^{4}=-\left(\left(\beta p+f_{, q}\right) \mathrm{d} q+f_{, p} \mathrm{~d} p\right) / \Delta,
\end{array}
$$


where

$$
\Delta=\alpha \beta p q+\alpha q f_{, q}+\beta p g_{, p}+\{f, g\} .
$$

We easily verify that

$$
\mathbf{e}_{1} \wedge \mathbf{e}_{2} \wedge \mathbf{e}_{3} \wedge \mathbf{e}_{4}=-\Delta \partial_{u} \wedge \partial_{v} \wedge \partial_{p} \wedge \partial_{q}
$$

and since the vector fields $\mathbf{e}_{i}$ should be independent we assume that $\Delta \neq 0$ holds. The above considerations are summarized in the following.

Proposition 4.1. Let the basis $\left\{\mathbf{e}_{i}\right\}$ given by (18), (19) be a null tetrad on $\mathbb{C}^{4}$ with coordinates $(u, v, p, q)$, and $\left\{\theta^{i}\right\}$ the dual basis. If $(U, P, R)$ satisfy equations (6)-(8) then the metric $g$ given by (13) is a representative of an $A S D$ conformal structure. If $\alpha=-\beta=1$ then there exists a conformal factor $\Lambda$ such that $\Lambda g$ is a ASD vacuum metric.

Proof. The first part is readily shown by the preceding construction. For the vacuum case, let $\nu=\mathrm{d} u \wedge \mathrm{d} v \wedge \mathrm{d} p \wedge \mathrm{d} q$ be the natural volume element on $\mathbb{C}^{4}$. By direct calculation we find that

$$
\begin{array}{ll}
\mathcal{L}_{\mathbf{e}_{1}} \nu=0, & \mathcal{L}_{\mathbf{e}_{3}} \nu=(\beta+1) \nu, \\
\mathcal{L}_{\mathbf{e}_{2}} \nu=0, & \mathcal{L}_{\mathbf{e}_{4}} \nu=-(\alpha-1) \nu,
\end{array}
$$

where $\mathcal{L}$ denotes the Lie derivative and $\nu\left(\mathbf{e}_{1}, \mathbf{e}_{2}, \mathbf{e}_{3}, \mathbf{e}_{4}\right)=-\Delta$. Hence, the vector fields $\mathbf{e}_{i}$ are divergence free with respect to $\nu$ whenever $\alpha=-\beta=1$. As such, the rescaled metric $-\Delta g$ has vanishing Ricci tensor.

It is worth noticing that when $\alpha=-\beta=1$ the hyperbolic Ernst equation is "switched off" 2 . Accordingly, using the above construction we may employ the plane wave solutions of the Ernst equation to construct, in principle, four dimensional ASD metrics but not vacuum ones.

\section{References}

1. F. Nijhoff, A. Hone and N. Joshi, Phys. Lett. A 267, 147 (2000).

2. A. Tongas, D. Tsoubelis and P. Xenitidis, J. Math. Phys. 42, 5762, (2001).

3. L. J. Mason and N.M.J. Woodhouse, Integrability Self-Duality, and Twistor Theory, LMS Monographs 15, Oxford Un. Press, Oxford, (1996).

4. R.S. Ward and R.O. Wells, Twistor geometry and field theory, Cambridge Monographs on Math. Phys., Cambridge Un. Press, Cambridge, (1990).

5. R. S. Ward, Phys. Lett. 61A, 81, (1977).

6. M. Ashtekar, T. Jacobson and L. Smolin, Commun. Math. Phys. 115, 631 (1988).

7. L.J. Mason and E.T. Newman, Commun. Math. Phys. 121, 659, (1989).

8. A. Tongas, D. Tsoubelis and P. Xenitidis, Phys. Lett. A 284, 266, (2001). 